\title{
Three-Dimensional Microfabricated Broadband Patch Antenna for WiGig Applications
}

\author{
H. Mopidevi, Student Member, IEEE, H. V. Hunerli, E. Cagatay, N. Biyikli, Member, IEEE, \\ M. Imbert, Student Member, IEEE, J. Romeu, Fellow, IEEE, L. Jofre, Fellow, IEEE, and
}

B. A. Cetiner, Member, IEEE

\begin{abstract}
The design, microfabrication, and characterization of a broadband patch antenna capable of covering the entire IEEE 802.11ad (WiGig) frequency band $(57-66 \mathrm{GHz})$ are presented in this letter. A conductor-backed (CB) coplanar waveguide (CPW)-fed loop slot couples the energy to the patch antenna, resulting in a broad bandwidth. The feed circuitry along with the loop is formed on a quartz substrate $\left(\varepsilon_{\mathrm{r}}=3.9, \tan \delta=0.0002\right.$ at $60 \mathrm{GHz}$ ), on top of which an SU-8-based three-dimensional (3-D) structure with air cavities is microfabricated. The patch metallization is deposited on top of this 3-D structure. While the main role of the structure made out of SU-8 material is to provide a mechanical support for the patch metallization, the antenna takes advantage of the air cavities underneath, thus resulting in an antenna substrate with a very low loss. This, in turn, improves the overall antenna performances. The simulated and measured impedance characteristics agree well, showing $\sim 15 \%$ bandwidth. Also, the radiation pattern results demonstrate the integrity of radiation pattern with reasonably constant gain values (average $\sim 6.4 \mathrm{~dB}$ ) in the broadside direction over the entire WiGig band.
\end{abstract}

Index Terms-Antenna measurements, coplanar waveguide (CPW)-fed patch antennas, IEEE 802.11ad, microfabrication, SU-8, W-band antennas, WiGig.

\section{INTRODUCTION}

A S THE ever-increasing demand for higher data rates continues, WiGig wireless technology $(57-66 \mathrm{GHz})$ enabling wireless data, voice, and video applications at multigigabit speeds has recently been attracting much interest in both academia and industry [1]. At millimeter ( $\mathrm{mm}$ )-wave frequencies ( $\sim 60 \mathrm{GHz}$ WiGig), the antenna design and manufacturing posses some challenges due mainly to the small dimensions, which may be as small as $\sim 20 \mu \mathrm{m}$, and lossy material characteristics, which are deleterious for antenna performances. Therefore, not only an effective microfabrication approach

Manuscript received February 18, 2014; revised April 03, 2014; accepted April 17, 2014. Date of publication April 22, 2014; date of current version May 06, 2014. This work was supported in part by the Center of Excellence Program of the Utah Governor's Office; the United States Department of Justice under Award No. 2009-SQ-B9-K005 awarded by the National Institute of Justice, Office of Justice Programs; and TUBITAK BIDEB.

H. Mopidevi and B. A. Cetiner are with the Department of Electrical and Computer Engineering, Utah State University, Logan, UT 84322-4120 USA (e-mail: swaroop.mopidevi@aggiemail.usu.edu; bedri.cetiner@usu.edu).

H. V. Hunerli, E. Cagatay, and N. Biyikli are with the National Nanotechnology Research Center (UNAM) and the Institute of Materials Science and Nanotechnology, Bilkent University, Ankara 06800, Turkey.

M. Imbert, J. Romeu, and L. Jofre are with the Universitat Politècnica de Catalunya, 08034 Barcelona, Spain (e-mail: marc.imbert@tsc.upc.edu).

Color versions of one or more of the figures in this letter are available online at http://ieeexplore.ieee.org.

Digital Object Identifier 10.1109/LAWP.2014.2319242 along with appropriate material use must be adopted, but also such considerations as being low-cost and compact and easy integration with transceiver must be taken into account.

Antennas operating at mm-wave frequencies have thus far mainly been implemented using either low temperature co-fired ceramic (LTCC) [2]-[4] or polymer substrates [5]. Although LTCC can create mechanically robust and hermetically sealed packages with high yield, it might create unwanted surface waves due to the high dielectric constant of substrate. Recently, planar antennas have also been realized on benzocyclobutene (BCB) polymers at $\mathrm{mm}$ waves [5]. $\mathrm{BCB}$ $\left(\varepsilon_{\mathrm{r}}=2.65, \tan \delta=0.0008\right)$, due to its electrical properties, is a good choice for improved antenna performance. However, BCB does not enable easy deposition as it requires multiple spins to achieve the desired thickness of a dielectric that could provide a reasonable operational bandwidth (BW) to a planar antenna within the IEEE 802.11ad band (57-66 GHz). Also, the very short shelf-life time of $\mathrm{BCB}$ under room temperature is another disadvantage. In order to create thick enough substrates, SU-8 $\left(\varepsilon_{\mathrm{r}}=3.1, \tan \delta=0.021\right)[6]$ is an excellent choice. However, its high loss tangent is the drawback for using it as a dielectric for planar antennas. Techniques such as creating holes, air cavities, etc., to reduce the effect of dielectric loss on the antenna performance exist in the literature [7], [8]. Combining the advantages of SU-8 along with these techniques yields an antenna substrate that is both electrically and mechanically an efficient solution. Hence, in this letter, microfabricated SU-8-based three-dimensional (3-D) structures with air cavities are used as low-loss alternative substrates for WiGig antennas.

Among the printed antennas, patch antennas allow for easier integration with RF front-end systems due to their compatibility with microwave integrated circuits. To enhance the BW of coplanar waveguide (CPW)-fed patches with omnidirectional radiation, a CPW-fed slot coupled patch approach is employed in [9]. To obtain a directional radiation pattern, a conductor backing is used for a CPW-fed slot antenna in [10]. Combining these two techniques, a conductor-backed (CB) CPW-fed loop slot (formed on quartz substrate) sourcing a microfabricated patch antenna on 3-D thick structural SU-8 layers is presented in this letter. This BW enhancement technique, which utilizes the quartz substrate for the loop metallization, is advantageous in simplifying the overall antenna architecture when compared to the stacked patch design [11], as the latter technique would need an additional layer for the stacked patch. The antenna design, in this letter, is carried out in conjunction with microfabrication process compatibility and mechanical integrity of the 3-D structure. The developed SU-8-based microfabrication process 


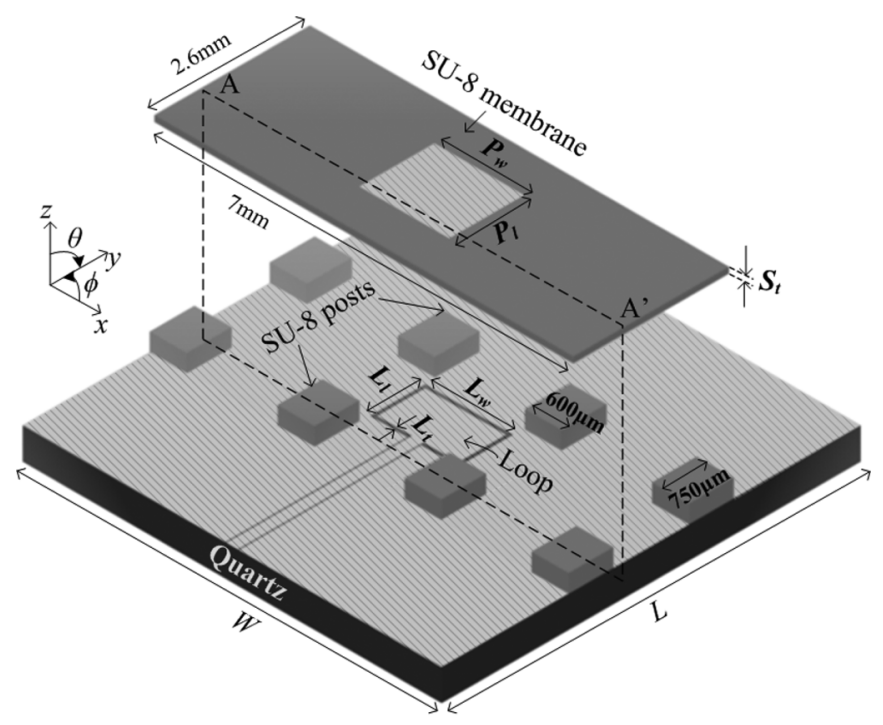

(a)

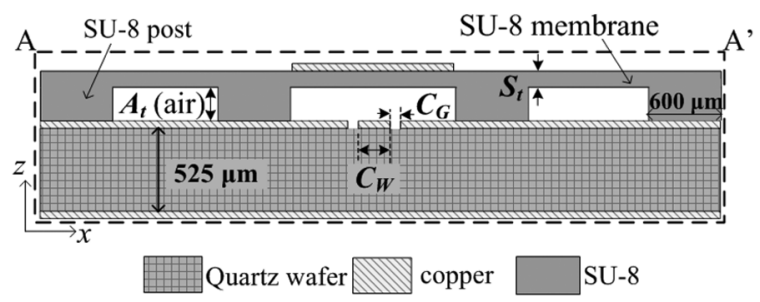

(b)

Fig. 1. Schematic depicting (a) 3-D (for the sake of illustration, the SU-8 membrane is suspended on top of the CPW metallization) and (b) cross-sectional drawings of the antenna.

presented in this letter can also be applied to reconfigurable antenna technologies for the next-generation WiGig systems.

\section{ANTENNA DESIGN}

The antenna as depicted in Fig. 1(a) and (b) is a CPW-fed broadband patch antenna microfabricated on an RF-compatible quartz substrate $\left(\varepsilon_{\mathrm{r}}=3.9, \tan \delta=0.0002\right.$ at $\left.60 \mathrm{GHz}\right)$. The feed metallization, which consists of a $50-\Omega$ CB CPW, along with the loop is formed on a $525-\mu \mathrm{m}$-thick quartz substrate. The SU-8-based 3-D substrate is microfabricated on top of the feed metallization as described in Section III. The 3-D substrate consists of an SU-8 membrane that is supported via SU-8 posts. The patch antenna metallization is finally formed on this 3-D substrate. The location of SU-8 posts and the thickness of SU-8 membrane $\left(S_{t}\right)$ dictate the mechanical stability of the 3-D antenna. As SU-8 is quite lossy, an intelligent design tradeoff between the mechanical integrity of the 3-D structure and the performance of the antenna needs to be incorporated. Accordingly, air cavities are incorporated in the 3-D SU-8 substrate to reduce the dielectric loss, which would in turn enhance the performance of the antenna. The height of the air cavity $\left(A_{t}\right)$, which is also the height of SU-8 posts, has an effect on the impedance BW and realized gain of the antenna.

To enhance the BW of the patch antenna, a CB CPW-fed rectangular loop slot (with dimensions $L_{\mathrm{l}}, L_{\mathrm{w}}$, and $L_{\mathrm{t}}$ ) couples the energy to the patch antenna. The resonant length of the loop is calculated as

$$
L_{\mathrm{l}}+L_{\mathrm{w}} / 2 \sim \lambda_{\mathrm{g}} / 2
$$

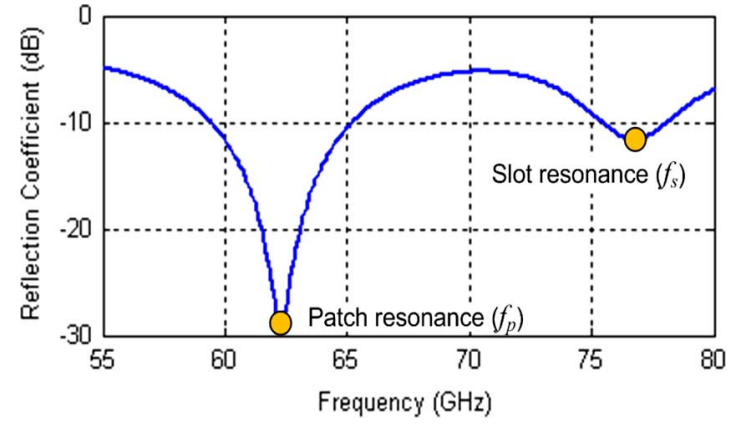

Fig. 2. Initial dual-band response of CB CPW-fed slot-loop coupled patch antenna with slot and patch resonances separated prior to design optimization.

where $\lambda_{\mathrm{g}}$ is the guide wavelength in quartz substrate at the resonant frequency $\left(f_{\mathrm{s}}\right)$. The substrate thickness of the CB CPW-fed loop slot plays an important role in broadening the radiation $\mathrm{BW}$ of the antenna. One of the main contributions of this letter is not only to improve the antenna performances in the WiGig band, but also to make the antenna design compatible with microfabrication processes, resulting in efficient and economic fabrication. Therefore, a $525-\mu \mathrm{m}$-thick quartz substrate, which is microfabrication-friendly as well as electrically thick to aid in broadband WiGig communication, is chosen. The patch antenna dimensions are calculated using [12]

$$
\begin{aligned}
& P_{1} \approx \frac{c}{2 f_{\mathrm{p}} \sqrt{\varepsilon_{\mathrm{r}}}} \\
& P_{1}<P_{\mathrm{w}}<2 P_{1}
\end{aligned}
$$

where $c$ is the speed of light; $f_{\mathrm{p}}$ is the resonant frequency, and $\varepsilon_{\mathrm{r}}$ is the effective dielectric constant. The initial dual-band response of the loop-slot coupled patch antenna is shown in Fig. 2, where the resonances at $f_{\mathrm{p}}=62.5 \mathrm{GHz}$ and $f_{\mathrm{s}}=77 \mathrm{GHz}$ correspond to patch and loop slots, respectively, according to (1) and (2). The parameters of CB CPW-fed loop and patch along with those of 3-D SU-8 substrate are jointly optimized to combine the patch and loop resonances, resulting in a broader BW. Also, the standard thickness of the microfabrication-compatible quartz wafer $(525 \mu \mathrm{m})$ is incorporated into the design optimization to attenuate possible surface waves. The height $\left(A_{\mathrm{t}}\right)$ and lateral area $\left(2.6 \times 2.6 \mathrm{~mm}^{2}-4 \times 0.75 \times 0.6 \mathrm{~mm}^{2}=4.96 \mathrm{~mm}^{2}\right)$ of air cavity beneath the patch, which suppresses the surface waves [8] and broadens the BW, can be precisely controlled within the realm of microfabrication feasibility using custom-made SU-8 3-D architectures. This methodology, taking the advantage of monolithically microfabricated 3-D structures, provides greater design flexibility and cost reduction when compared to etching away portions of bulky substrates or creating vias/holes in substrates with standard thickness [7], [8].

The optimized design parameters of the patch element, CPW-fed loop, and the 3-D substrate obtained from full-wave simulation are provided in Table I. Using these optimized parameters and (2), the effective dielectric constant $\left(\varepsilon_{\mathrm{r}}\right)$ of the SU-8-based 3-D structure including quartz wafer is verified to be $\sim 3.8$, which is in between that of quartz $\left(\varepsilon_{\mathrm{r}}=3.9\right)$ and SU-8 $\left(\varepsilon_{\mathrm{r}} \sim 3.1\right)$. This design methodology not only minimizes the dielectric loss of SU-8 through air cavities, but also results in compact antenna size due to higher effective dielectric constant. Secondly, the patch metallization on top of the SU-8 
TABLE 1

Critical Design Parameters of the 3-D Wigig Antenna (All Dimensions ARE IN MiLlimeters)

\begin{tabular}{|c|c|c|c|c|c|c|c|}
\hline$W$ & 7 & $P_{w}$ & 1.5 & $L_{l}$ & 0.92 & $C_{G}$ & 0.02 \\
\hline$L$ & 7 & $P_{l}$ & 1.25 & $L_{w}$ & 1.47 & $C_{W}$ & 0.191 \\
\hline$L_{t}$ & 0.06 & $S_{t}$ & 0.1 & $A_{t}$ & 0.2 & \multicolumn{1}{|c}{} \\
\hline
\end{tabular}

substrate focuses the electromagnetic (EM) energy to result in a narrower beamwidth, which is otherwise broader for a standard CPW-fed loop. Finally, the patch metallization also decreases the cross-polarization level to at least $16 \mathrm{~dB}$ (as opposed to $\sim 9 \mathrm{~dB}$ in a standard CPW-fed loop) below the copolarization level of the presented antenna as shown in Section IV.

\section{MICROFABRICATION}

A four-mask-level, microwave-compatible microfabrication process is developed utilizing 3-D thick SU-8 coating and patterning processes as well as standard thin-film deposition and patterning/etching processes. Prior to fabrication, 4-in RF-compatible/low-loss quartz substrates $\left(\varepsilon_{\mathrm{r}}=3.9, \tan \delta=0.0002\right.$ at $60 \mathrm{GHz}$ ) were cleaned using standard acid/solvent cleaning, DI-water rinsing, nitrogen-blow drying, and dehydration baking on a $120^{\circ} \mathrm{C}$ hotplate.

As summarized schematically in Fig. 3(a), the fabrication process started with: 1) physical vapor deposition (PVD) and patterning of the CPW metal layer that was formed of titanium/copper $(\mathrm{Ti} / \mathrm{Cu})$ where Ti functioned as the 10 -nm-thick adhesion layer and $\mathrm{Cu}$ thickness was $\sim 300 \mathrm{~nm}$. Sputtering and thermal evaporation were the most dominantly used PVD methods, whereas the patterning was realized using either a liftoff or wet metal-etch process. After the metallic CPW was formed: 2) blank $\mathrm{Ti} / \mathrm{Cu}$ deposition on the backside of quartz wafer was implemented, which functioned as the ground metal layer. 3) Later, SU-8 coating and patterning process consisting of two mask layers was carried out. A 300- $\mu$ m-thick SU-8 layer was reached using two consecutive spin coatings of $150-\mu \mathrm{m}$ layers and soft-baked at $95^{\circ} \mathrm{C}$ for 45 and $90 \mathrm{~min}$ after each layer deposition, which was followed by two separate exposures. The first (second in the overall process) mask was used to cross-link the post regions for a total dose of $300 \mathrm{~mJ} / \mathrm{cm}^{2}$. A lower exposure dose $\left(95 \mathrm{~mJ} / \mathrm{cm}^{2}\right)$ was used in the second (third mask in the overall process) mask with the aim of cross-linking only the $100-\mu \mathrm{m}$ upper layer of the membrane areas. Immediately after the exposure steps, a 3 -min post-exposure bake (PEB) at $55^{\circ} \mathrm{C}$ was applied with sufficient ramp-up and ramp-down durations to minimize the stress accumulation within the structural SU-8 layers. 4) Patch metal deposition and patterning followed using $\sim 300$-nm-thick $\mathrm{Ti} / \mathrm{Cu} \mathrm{DC}$-sputtering and wet-etch patterning using the fourth lithography mask. Finally: 5) the sample is soaked into SU-8 developer solution within an ultrasonic bath, targeting to dissolve and remove the uncross-linked SU-8 layer underneath the partially exposed membrane.

Seven to $8 \mathrm{~min}$ of agitated development process was enough to completely remove the unprocessed SU-8 material, and the membranes were released successfully. The microfabrication process of our 3-D WiGig antenna was completed with a hard baking process of the device over a hotplate at $110{ }^{\circ} \mathrm{C}$

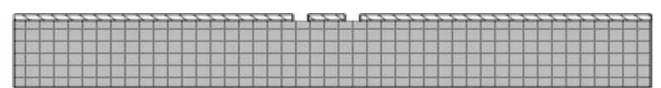

(1)

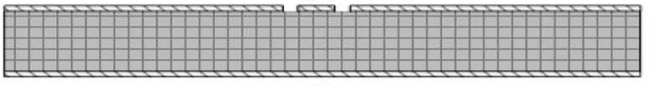

(2)

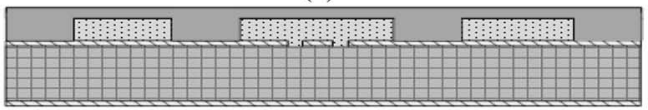

(3)



(4)

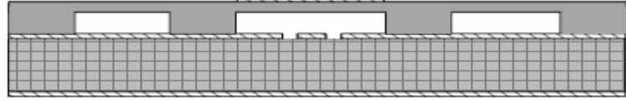

(5)

Uncross-linked SU-8

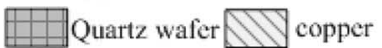
SU-8

(a)

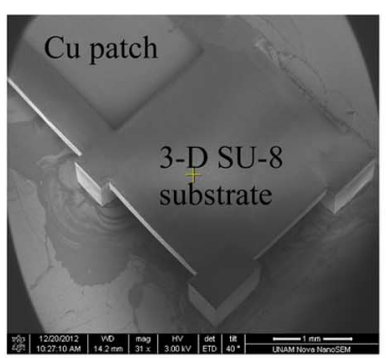

(b)

Fig. 3. (a) Cross-sectional schematic representation of the four-mask-level SU-8-based 3-D microfabrication process flow. (b) Angled-view SEM micrograph of a completed antenna device with SU-8 posts and fully released SU-8 membrane along with patterned $\mathrm{Cu}$ patch metal layer on top.

to strengthen the structure by further cross-linking the SU-8 layers. Fig. 3(b) shows a scanning electron microscope (SEM) photograph of a completed device.

\section{MEASUREments AND CHARACTERIZATION}

The performance of the microfabricated loop-coupled patch antenna with $\mathrm{CPW}$ feed has been measured with an Agilent $8510 \mathrm{C}$ vector network analyzer (VNA) together with a GSGprobe station from 50 to $67 \mathrm{GHz}$.

The pitch of the probes (distance between one ground tip and the signal tip) is of $150 \mu \mathrm{m}$. The CPW feeding lines of the patch antenna were designed to perfectly accommodate to this probe pitch. One port calibration process was performed manually, measuring three known standards (i.e., open, short, and broadband matched load terminations) in order to establish the measurement reference planes. The wafer with the microfabricated patch antenna prototypes was placed over the sample stage. Since the antenna was designed with a CB CPW feed, the effect of the metal plate of sample stage in the measurement setup has been minimized. The sample stage has a movable metal plate that allows probes to contact anywhere on the wafer surface with precise motion in a 2-D plane ( $x y$-directions). The stage is equipped with a vacuum chuck to fix the wafer to the metal plate, with the aim to ensure the perfect contact of the 


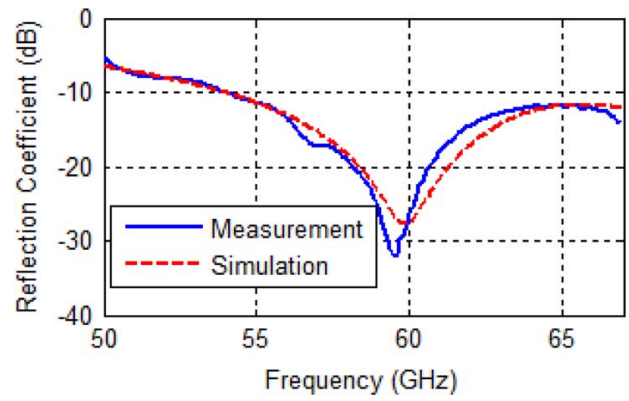

Fig. 4. Simulated and measured magnitudes of $S_{11}$ parameter (reflection coefficient) for a frequency range from 50 to $67 \mathrm{GHz}$ obtained for the microfabricated broadband patch antenna.

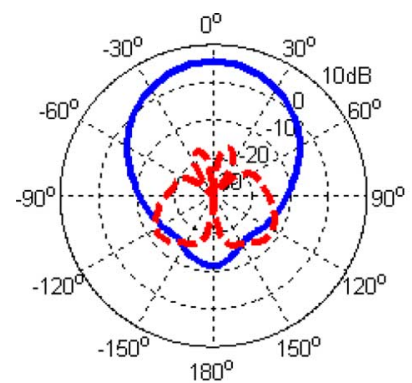

(a)

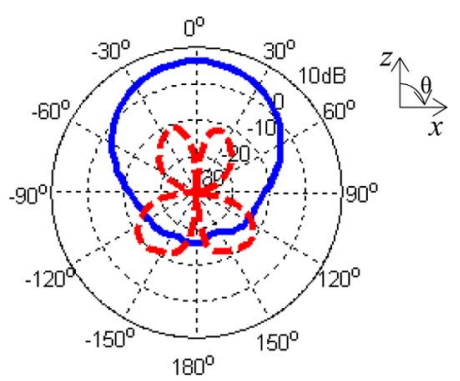

(b)

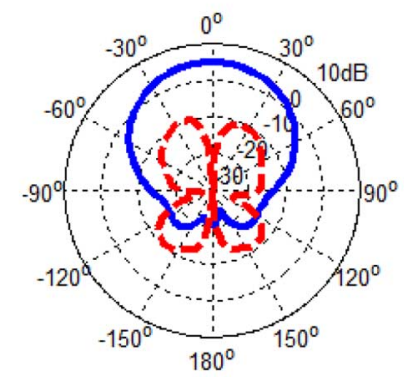

(c)

Fig. 5. Simulated realized gain plot (dB) of the antenna in $x z$-plane at (a) 57 (b) 62 , and (c) $66 \mathrm{GHz}$.

probe tips with the CPW feeding the patch antenna. A PC communicates with the VNA in order to control the process, run the calibration software, and store the measurements.

The simulated and measured reflection coefficients, with good agreement between them, are plotted in Fig. 4 for a frequency range from 50 to $67 \mathrm{GHz}$. Slight variation between measured and simulated results can mainly be attributed to the fabrication tolerances. The reflection coefficient shows that the antenna has a 2:1 VSWR BW of greater than $9 \mathrm{GHz}(\sim 15 \%$ of fractional $\mathrm{BW}$ ), which covers the entire frequency range of the IEEE 802.11ad (57-66 GHz). The simulated radiation patterns of the linearly polarized antenna in $x z$-plane at 57,62 , and $66 \mathrm{GHz}$ are shown in Fig. 5(a)-(c), respectively, showing a patch-type pattern, as expected (at the time of this work, we did not have the capability of performing radiation pattern measurements at $60 \mathrm{GHz}$ ). The cross-polarization level is at least $16 \mathrm{~dB}$ below the copolarization level of the antenna. The realized maximum gain of the antenna is relatively constant and is in the range $\sim 5.5-7 \mathrm{~dB}$ over the entire $\mathrm{BW}$ as shown in Fig. 6. Higher gain is obtained due to the presence of air

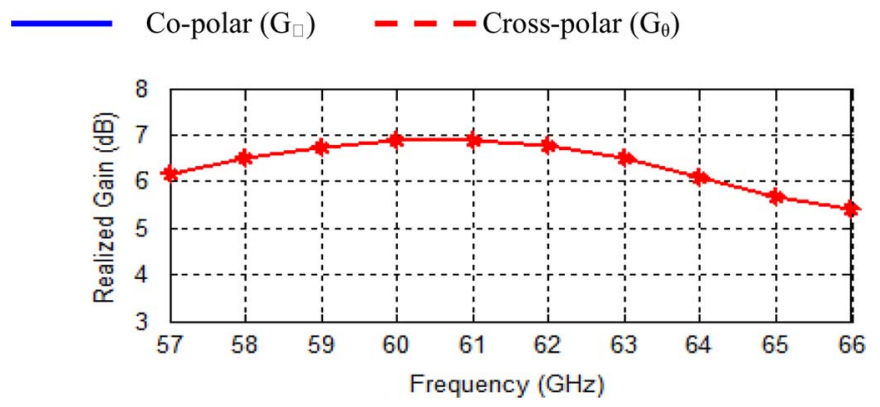

Fig. 6. Simulated realized gain $(\mathrm{dB})$ in the broadside direction of the antenna with respect to frequency.

cavities in the 3-D SU-8 substrate, which effectively reduces the dielectric loss.

\section{CONCLUSION}

A CPW-fed broadband patch antenna compatible with IEEE 802.11ad standard (WiGig) is designed, microfabricated, and characterized. The measured reflection coefficient data is in good agreement with the simulation giving $\sim 15 \% \mathrm{BW}$. The simulated radiation patterns with reasonably constant gain values $(5.5-7 \mathrm{~dB})$ in the broadside direction over the entire WiGig band (57-66 GHz) indicate a design with low dielectric loss. The SU-8-based 3-D microfabrication processes developed for this antenna structure provides an important advantage for custom-made reconfigurable antennas that might also be greatly useful in WiGig applications.

\section{REFERENCES}

[1] C. J. Hansen, "WiGig: Multi-gigabit wireless communications in the $60 \mathrm{GHz}$ band," IEEE Wireless Commun., vol. 18, no. 6, pp. 6-7, Dec. 2011.

[2] T. Seki, N. Honma, K. Nishikawa, and K. Tsunekawa, “A 60-GHz multilayer parasitic microstrip array antenna on LTCC substrate for system-on-package," IEEE Microw. Wireless Compon. Lett., vol. 15, no. 5, pp. 339-341, May 2005.

[3] Y. P. Zhang, M. Sun, K. M. Chua, L. L. Wai, D. Liu, and B. P. Gaucher, "Antenna-in-package in LTCC for 60-GHz radio," in Proc. IEEE Int. Workshop Antenna Technol., Mar. 2007, pp. 279-282.

[4] S. Wi et al., "Package level integrated antennas based on LTCC technology," IEEE Trans. Antennas Propag., vol. 54, no. 8, pp. 2190-2197, Aug. 2006.

[5] S. Seok;, N. Rolland, and P.-A. Rolland, "Millimeter-wave quarterwave patch antenna on benzocyclobutene polymer," in Proc. 38th Eur. Microw. Conf., Oct. 27-31, 2008, pp. 1018-1021.

[6] A.-D. Campo and C. Greiner, "SU-8: a photoresist for high-aspect-ratio and 3D submicron lithography," J. Micromech. Microeng., vol. 17, pp. R81-R95, 2007.

[7] G. P. Gauthier, A. Courtay, and G. M. Rebeiz, "Microstrip antennas on synthesized low dielectric-constant substrates," IEEE Trans. Antennas Propag., vol. 45, no. 8, pp. 1310-1314, Aug. 1997.

[8] D. R. Jackson, J. T. Williams, and A. K. Bhattacharyya, "Microstrip patch designs that do not excite surface waves," IEEE Trans. Antennas Propag., vol. 41, no. 8, pp. 1026-1037, Aug. 1993.

[9] S.-W. Lu, T.-F. Huang, and P. Hsu, "CPW-fed slot-loop coupled patch antenna on narrow substrate," Electron. Lett., vol. 35, no. 9, pp. 682-683, Apr. 29, 1999.

[10] H. C. Liu, T. S. Horng, and N. G. Alexopoulos, "Radiation from aperture antennas with a coplanar waveguide feed," in IEEE AP-S Symp. Digital Archive, 1992, pp. 1820-1823.

[11] R. Q. Lee, K. F. Lee, and J. Bobinchak, "Characteristics of a twolayer electromagnetically coupled rectangular patch antenna," Electron. Lett., vol. 23, no. 20, pp. 1070-1072, Sep. 24, 1987.

[12] P. Bhartia, I. Rahl, R. Garg, and A. Ittipiboon, Microstrip Antenna Design Handbook. Norwood, MA, USA: Artech House, 2001. 